\title{
Comments by Author regarding: Levy, G.S. Thermoelectric Effects under Adiabatic Conditions. Entropy 2013, 15, 4700-4715
}

\author{
George Levy \\ Received: 24 November 2015; Accepted: 16 December 2015; Published: 17 December 2015 \\ Academic Editor: Kevin H. Knuth \\ Entropic Power Corporation, 3980 Del Mar Meadows, San Diego, CA 92130, USA; \\ glevy@entropicpower.com; Tel.: +1-858-259-2226; Fax: +1-858-259-2233
}

\begin{abstract}
This comment by the author discusses paragraph 3.3. Adiabatic Phenomena-An Analogy of the published article [1]. The paragraph draws an incorrect analogy between Maxwellian gases such as found in the atmosphere, and non-Maxwellian gases embodied, for example, by electrical carriers in thermoelectric materials. As reported by the author in more recent articles, Maxwellian gases do not produce spontaneous temperature gradients in the presence of a force field. However, non-Maxwellian gases, such as Fermions or Bosons, can produce such gradients.
\end{abstract}

The example given in paragraph 3.3. Adiabatic Phenomena-An Analogy of the article is not a good representation of the thermoelectric effect under discussion because a Maxwellian gas such as found in the atmosphere does not produce any spontaneous temperature gradient. A better treatment is given by the author in $[2,3]$.

The article correctly mentions that Maxwellian molecules in a gas column lose energy and become less dense as they move upward in a gravitational field. Surprisingly however, their energy distribution does not change with elevation implying that the gas is isothermal. The reason is that gravity operates on molecules as an energy separator (in analogy to a mass spectrometer) allowing those with a higher total energy to move up and those with lower energy to stay down. Those with the higher total energy (kinetic + potential) are found at higher elevation simply because they start out with more total energy. Molecules get vertically separated according to their total energies. In a Maxwellian gas this separation effect cancels out exactly the kinetic energy lost by rising molecules. Therefore, the gas shifts towards an isothermal state as it moves toward equilibrium in conformance with the Second Law. Another explanation is that in Maxwellian gases, potential energy is expressed within the exponential term of the distribution. Therefore a change in altitude, while causing a change in density, can be factored out of the exponential term by renormalizing the distribution as shown in $[2,3]$ thereby eliminating the effect of elevation.

In these more recent articles, the author shows that a temperature gradient is produced spontaneously only if the gas is comprised of non-Maxwellian particles such as Fermions or Bosons. Their energy distribution is not strictly exponential and therefore, renormalization does not eliminate the potential energy term. They develop a temperature gradient when subjected to an electric field as correctly described in the article and in [2,3]. In addition, the Monte-Carlo simulator mentioned in the article does produce a temperature gradient because the distribution imparted to the gas particles is an impulse (non-Maxwellian), as they collide with the walls, floor and ceiling of the simulation chamber.

In [2] the author shows that the same effect can be used to shift the odds in a two-coin flipping game. When the coins are classical, the outcomes, (Head-Head), (Head-Tail), (Tail-Head), (Tail-Tail) are evenly distributed. However, when the coins are Fermions and located in an energy well, the Pauli Exclusion Principle shifts the odds in favor of (Head-Tail), (Tail-Head) when the coins are selected 
from the same energy level at the bottom of the well. The coin game and the non-Maxwellian gas column utilize the same environment: a Fermion gas in a column subjected to a force field. The Pauli Exclusion Principle shifts the odds in the coin game. It also shifts the kinetic energy in the non-Maxwellian gas column, resulting in a spontaneous temperature gradient. The same phenomenon also applies to Bosons.

Electrical carriers in a semiconductor are Fermions and, therefore, they can develop a temperature gradient in the absence of any electrical current when exposed to an electrical field as correctly described by the article. In ordinary semiconductors this temperature gradient is not observable but can be measured in high ZT materials characterized by a low coupling between electrical carriers and heat phonons.

Conflicts of Interest: The author declares no conflict of interest.

\section{References}

1. Levy, G.S. Thermoelectric Effects under Adiabatic Conditions. Entropy 2013, 15, 4700-4715. [CrossRef]

2. Levy, G.S. Quantum Game Beats Classical Odds-Thermodynamics Implications. Entropy 2015, 17, 7645-7657. [CrossRef]

3. Levy, G.S. Anomalous Temperature Gradient in Non-Maxwellian Gases. In Proceedings of the 11th International Conference on Ceramic Materials \& Components for Energy \& Environmental Applications, Vancouver, BC, Canada, 14-19 June 2015; Wiley: Hoboken, NJ, USA, 2016; Volume 255, in press.

(C) 2015 by the author; licensee MDPI, Basel, Switzerland. This article is an open access article distributed under the terms and conditions of the Creative Commons by Attribution (CC-BY) license (http://creativecommons.org/licenses/by/4.0/). 